\title{
Changes in the Autonomic Nervous System and Moods of Advanced Cancer Patients by Mindfulness Art Therapy Short Version
}

\author{
Michiyo Ando ${ }^{*}$, Haruko Kira ${ }^{2}$, Shigeru Hayashida ${ }^{3}$ \\ ${ }^{1}$ Faculty of Nursing, St. Mary's College, Fukuoka, Japan \\ ${ }^{2}$ Institute of Comparative Studies of International Cultures and Societies, Kurume University, Fukuoka, Japan \\ ${ }^{3}$ Naito Hospital, Kurume, Japan \\ Email: *andou@st-mary.ac.jp
}

Received 6 November 2015; accepted 8 January 2016; published 11 January 2016

Copyright (C) 2016 by authors and Scientific Research Publishing Inc.

This work is licensed under the Creative Commons Attribution International License (CC BY).

http://creativecommons.org/licenses/by/4.0/

(c) (i) Open Access

\begin{abstract}
The aim of this study was to investigate changes in the Autonomic Nervous System (ANS) and moods before and after participation in a Mindfulness Art Therapy Short version (MATS). The study design was non-randomized controlled trial in intervention study. Participants were 10 Japanese patients with advanced cancer. They received the MATS in one session, which consisted of mindfulness practices and making arts. Their Autonomic Nervous System (sympathetic nervous, parasympathetic nervous) physiologically and mood (Tense Arousal: TA, Energetic Arousal: EA) psychologically were measured before and after the MATS. The results showed that the level of parasympathetic nervous system decreased and that the sympathetic nervous system increased in small effect size. The TA decreased in a large effect size and EA increased in a middle effect size. These results suggested that the MATS might activate physiologically, alleviate tension and increase energy psychologically.
\end{abstract}

\section{Keywords}

Mindfulness, Art Therapy, Automatic Nervous System, Mood, Cancer Patients

\section{Introduction}

Cancer patients experience a number of physical and psychological problems. Art therapy is a type of psychotherapy which can be effective in the alleviation of anxiety, depression and somatic symptoms [1]. Mindfulness

${ }^{*}$ Corresponding author.

How to cite this paper: Ando, M., Kira, H. and Hayashida, S. (2016) Changes in the Autonomic Nervous System and Moods of Advanced Cancer Patients by Mindfulness Art Therapy Short Version. Journal of Cancer Therapy, 7, 13-16. 
is another effective approach. Kabat-Zin [2] develops the Mindfulness-Based Stress Reduction (MBSR) program, which is based on the principle of mindfulness, defined as moment-to-moment, present-centered, purposive non-judgmental awareness. Mindfulness is effective on psychological status, physical status [3].

Monti et al. [4] develop Mindfulness-Based Art Therapy (MBAT) which integrates mindfulness and art therapy, and provides significant decreases to the symptoms of distress and increases in measurements of quality of life. Physiologically, undergoing MBAT for 8 weeks increased CBF (Cerebral Blood Flow) and a correlation is found between increased CBF and decreased anxiety [5].

Although the duration of the MBAT is around 8 weeks, and the intervention is performed in a group setting, this approach may be difficult for patients who are hospitalized with advanced cancer. To address this, Ando, Kira, \& Hayashida [6] develop a simpler version, they call "The Mindfulness Art Therapy Short version (MATS)". The MATS includes mindfulness and art therapy in two sessions. Ando et al. conducts the MATS with patients suffering from advanced cancer, and the results suggest that the method may be effective at increasing energy and reducing fatigue [6].

However, it is not clear precisely what physiological change is caused by the MATS. One of the physiological indicators is the Autonomic Nervous System (ANS). ANS reflects Heart Rate Variability (HRV), which provides a look at the delicate relationship between the heart and lungs. Porges [7] says that brain scan and EEG studies require immobility, and an action-based form of mindfulness or therapeutic use of the arts are not possible, however, resting HRV has become an accepted marker for vagal tone and neurobiological correlations of internal composure. Thus, we investigate changes in the automatic nervous system as the physiological response and moods as the psychological response.

\section{Method}

Study Design: The study design was non-randomized controlled trial in intervention study.

Setting: The study was conducted in western Japan. Period of patients' recruitment and data collection was from April 2013 to May 2014.

\subsection{Participants}

Participants were 10 patients with advanced cancer in a general hospital in Japan (male; 2, female: 8). A range of ages was from 38 to 72 years old and the mean age was 53.6. All patients were married except one. All patients were suffering from metastatic, stage IV cancer. They had a variety of types of cancer. The inclusion criteria were patients aged over 20 years old who could communicate easily and gave informed consent to participate in the study.

\subsection{Measurements}

Autonomic Nervous System: we used the TAS 9 (YKC Cooperation), an instrument for measuring heart rates, to measure total energy levels (TP), both sympathetic and parasympathetic nervous level (LF: low frequency), parasympathetic nervous level (HF: high frequency), the balance of sympathetic and parasympathetic nervous levels, as well as the sympathetic nervous system (LF/HF).

Moods: we used the Japanese UWIST Mood Adjective Checklist (JUMACLE) [8]. It consisted of tense arousal (TA) which includes tension or anxiety and energetic arousal (EA) which includes activity or vigor. It consisted of 10 TA and 10 EA items. Participants measure each item from 1 to 4 on the Likert scale. High scores represent high tension arousal or energetic arousal.

\subsection{Mindfulness Art Therapy Short Version Program}

The intervention consists of mindfulness exercises and art therapy for 90 minutes. We conducted the intervention two times [6], however, for this study we only performed one session because we examined physiological and psychological changes prior to and after the intervention. Patients are instructed on mindfulness with the assistance of a CD developed by a Yoga specialist and a clinical psychologist. We have previously shown the feasibility of this approach. Firstly, patients learned mindfulness by listening to the CD from the clinical psychologist. This instruction required about 20 minutes. The simple instructions on the CD were designed to make the patients aware of their minds and bodies without judgment. 
After mindfulness practice, patients were provided with materials including clay, items to make a collage (fancy paper, felt glue sticks, magazines), drawing instruments (colored pencils, pastel chalks, pencils, water colors), and sketch books. Patients were invited to make art "Please express your feelings or emotions freely".

\subsection{Procedure}

This study was approved by the ethical board of St. Mary's College. The physician in charge of a general hospital in Japan recruited patients with advanced cancer and obtained informed consent after explaining the study to them. If a patient agreed to participate in the study, the physician called the clinical psychologist. She visited the patients and explained the study in more detail. If the patients continued to agree to participate, they signed an informed consent form. A day after the informed agreement, the clinical psychologist conducted the MATS for about 90 minutes. ANS was measured using the TAS 9 by putting the sensor on the figure and participants completed JUMACLE before and after the intervention.

Data analysis: The physician explained the study to patients who were clear the criteria to protect bias about selection of participants. As for study size, it is difficult patients with advanced cancer to complete both mindfulness and art therapy including measurement, we set 10 patients. Thus we used effect size to examine effect of the intervention. Based on published criteria [9], an effect size of from 0.2 to $<0.5,0.5$ to $<0.8$, and $>0.8$ were defined as small, medium, and large, respectively.

\section{Results}

The mean scores of the measurements of the ANS and moods are shown in Table 1.

Since the number of participants was small, we used the effect size to examine the differences before and after the therapy. In evaluating the data, we referred to this criteria. There was a small decrease in HF (4.19 pre-intervention to 3.77 post-intervention), and increases in LF/HF (1.24 pre, and 1.32 post). There were no effects on $\mathrm{TP}(6.68,6.63)$ and LF $(4.92,4.94)$. As for mood as psychological indicator, there was big decrease in TA (17.5, $14.8)$, and a middle increase in effect EA $(26.1,28.7)$.

\section{Discussion}

Regarding the basic data on ANS, HF, LF, and LF/HF scores, they are within the standard range. However, the TP score is out of the standard range. This suggests that the level of ANS in cancer patients is critically low and care is required to promote it. During life-threatening situations and other challenges which require high-energy output, SNS activation of the stress response is necessary [10]. Thus, to continue to cope with the worsening cancer of ANS may be needed.

The results that HF scores decrease and LF/HF scores increase after the MATS, though they are small effect size, suggest that patients' sympathetic nervous system may have been activated. That is, this therapy may activate cancer patients and increase their physiological vigor.

The reasons why vigor increased will now be explained. Previous studies demonstrated that meditation increases CBF [11] as well as theta and alpha Electroencephalogram (EEG) activity [12]. Then mindfulness, including meditation during the MATS, physiologically promotes brain activity. Moreover, a previous study in the literature about art therapy, single-case EEG studies of art-perception, and making art suggests bi-lateral hemispheric activity [13]. This study suggests that making arts promote activity. Thus, the MATS including both mindfulness and art therapy activates the sympathetic nervous system and increases vigor. These activated effects

Table 1. Scores of ANS pre-and post-therapy $(\mathrm{N}=10)$.
\begin{tabular}{|cccccc}
\hline & Pre & Post & Effect size & Size & Standard Range \\
\hline HF & $4.19 \pm 2.26$ & $3.77 \pm 1.72$ & -0.35 & Small & $4.00-7.23$ \\
LF/HF & $1.24 \pm 0.36$ & $1.32 \pm 0.28$ & -0.36 & Small & $0.52-2.32$ \\
TP & $6.68 \pm 1.16$ & $6.63 \pm 0.83$ & -0.084 & None & $7.26-9.17$ \\
LF & $4.92 \pm 2.10$ & $4.94 \pm 2.00$ & 0.78 & None & $6.00-8.06$ \\
TA (Tense Arousal) & $17.5 \pm 7.10$ & $14.8 \pm 7.52$ & -0.55 & Large & $10-40$ \\
EA (Energetic Arousal) & $26.1 \pm 8.61$ & $28.7 \pm 9.17$ & -0.45 & Middle & $10-40$ \\
\hline
\end{tabular}


support previous study [6] in which this therapy promotes vigor in mood. Moreover, increases in vigor ae similar to the degree that MBAT increases CBF in a study by Monti et al. [4].

Psychologically, the TA score decreases in large effect size. Participants may be able to calm themselves through mindfulness. A previous study reports that the incorporation of mindfulness and meditation into psychotherapy can contribute to the repair of insecure attachment, interpersonal emotional dysregulation, and destructive coping [14]. The capacity to be aware of one's current experience leads to emotional well-being [15]. Thus, TA may decrease. On the other hand, EA increases in the middle effect size. Since participants can concentrate on their own mind, they can easily express their feelings or thoughts freely, possibly accounting for the increase in EA.

Lastly, regarding limitations of the present study, the number of participants is small. To confirm these results, we need to increase the number of participants.

\section{Funding}

This study was supported by JSPS KAKENHI Grant Number 245309010001.

\section{References}

[1] Thyme, K.E., Sundin, E.C. and Wiberg, B. (2009) Individual Brief Art Therapy Can Be Helpful for Women with Breast Cancer: A Randomized Controlled Clinical Study. Palliat Support Care, 7, 87-95. http://dx.doi.org/10.1017/S147895150900011X

[2] Kabat-Zinn, J. (1990) Full Catastrophe Living: Using the Wisdom of Your Body and Mind to Face Stress, Pain and Illness. Delacourt, New York.

[3] Langacher, C.A., Kip, K.E., Barta, M., et al. (2012) A Pilot Study Evaluation the Effect of Mindfulness-Based Stress Reduction on Psychological Status, Physical Status, Salivary Cortisol, and Interleukin-6 among Advanced-Stage Cancer Patients and Their Caregiver. Journal of Holistic Nursing, 30, 170-185. http://dx.doi.org/10.1177/0898010111435949

[4] Monti, D.A., Peterson, C. and Kunkel, S. (2006) A Randomized, Controlled Trial of Mindfulness-Based Art Therapy (MBAT) for Women with Cancer. Psycho-Oncology, 15, 363-373. http://dx.doi.org/10.1002/pon.988

[5] Monti, D.A., Kash, K.M. and Kunkel, E.J., et al. (2012) Changes in Cerebral Blood Flow and Anxiety Associated with an 8-Week Mindfulness Program in Women with Breast Cancer. Stress \& Health, 28, 397-407. http://dx.doi.org/10.1002/smi.2470

[6] Ando, M., Kira, H. and Hayashida, Y. (2016) Potential Effect of Mindfulness Art Therapy Short Version on Mood in Patients with Advanced Cancer. Art Therapy: Journal of the American Art Therapy Association, in press.

[7] Porges, S.W. (2011) The Polyvagal Theory: Neurophysiological Foundations of Emotions, Attachment, Communication, Self-Regulation. Norton, New York.

[8] Shirasawa, S., Ishida, T., Hakoda, Y., Haraguchi, M. (1999) The Effects of Energetic Arousal on Memory Search. The Japanese Journal Psychonomic Science, 17, 93-99.

[9] Cohen, J. (1988) Statistical Power Analysis for the Behavioral Sciences. 2nd Edition, Lawrence Erlbaum, Hillsdale.

[10] Rappaport, L. (2014) Mindfulness and the Arts Therapies. Jessica Kngsley Publisher, London, 294.

[11] Newberg, A.B., Wintering, N., Waldman, M.R., et al. (2010) Cerebral Blood Flow Differences between Ling-Term Meditators and Non-Meditators. Consciousness and Cognition, 19, 899-905. http://dx.doi.org/10.1016/j.concog.2010.05.003

[12] Lagopoulos, J., Xu, J., Rasmussen, I., et al. (2009) Increased Theta and Alpha EEG Activity during Nondirective Meditation. Journal of Alternative and Complementary Medicine, 15, 1187-1192. http://dx.doi.org/10.1089/acm.2009.0113

[13] Belkofer, C.M. and Konopka, L.M. (2008) Conducting Art Therapy Research Using Quantitative EEG Measures. Art Therapy: Journal of the American Art Therapy Association, 25, 56-63. http://dx.doi.org/10.1080/07421656.2008.10129412

[14] Germer, C.K. and Siegel, R.D. (2012) Wisdom and Compassion in Psychotherapy: Deepening Mindfulness in Clinical Practice. Guilford, New York.

[15] Siegel, D.J. (2007) The Mindful Brain: Reflection and Attunement in the Cultivation of Well-Being. New York, Norton. 